\title{
An inexpensive videoprojection/digitization system for behavioral research
}

\author{
MATTHEW K. LITVAK and ROGER I. C. HANSELL \\ University of Toronto, Toronto, Ontario, Canada
}

\begin{abstract}
We present an inexpensive approach to image analysis of spatial coordinates for behavioral research. Image analysis of still video images (freeze frames) with current technology can be prohibitively expensive, as well as time consuming. The system outlined here provides an accurate, inexpensive, and fast approach to acquiring information from videotape. It consists simply of a video monitor, bellows, large diameter lens, and a digitizing tablet. The images are projected onto a digitizing tablet, and data acquisition is controlled with a series of three functions written in APL. We used this system to acquire positional information on fish responding to the threat of aerial predation. We discuss several approaches to design and construction of this system, as well as provide computer listings for initiation of communication with digitization boards.
\end{abstract}

Behavioral research has been greatly enhanced by current "high-tech" advances in video and computer technology. Videotaping of behavior is not only inexpensive, it now offers many affordable advanced features, such as low light videotaping ( 1 lux), videotaping under a wide variety of light spectra (e.g., UV); high resolution; time/ date generators; clear freeze frame; advanced editing features; high shutter speed; and so forth. The advanced features provided by current videographic systems are ideal for behavioral analysis of spatial relationships between animals (or parts of animals).

Coordinates from video images can be captured in a variety of different ways. For example, computer image analysis, incorporating the use of a frame grabbing board in conjunction with a software package, will satisfy many analytic requirements of the researcher. However, these systems can be prohibitively expensive; a good frame grabber costs in excess of $\$ 2,000$, and software starts at $\$ 500$. It is not uncommon to spend $\$ 5,000$ on software alone. In addition, these systems are geared to analyze only images captured from a moving frame. To acquire a paused (freeze-frame) image in this manner, the source (VCR) must be synchronized with the image board in the computer. Images that are paused with VCRs that do not have digital freeze-frame capabilities experience jitter. This jitter results from an inconsistent H-Sync signal, caused by the playback heads being passed continually over one section of the videotape. A VCR with a separate chip to digitally encode the frozen image is required in

We thank D. J. Hamilton, M. M. Litvak, and J. Schmidt for their help with this work. This research was supported by Natural Sciences and Engineering Research Grants to R.I.C.H., who is in the Department of Zoology at Toronto, and to M.K.L., to whom correspondence may be addressed at the Huntsman Marine Science Centre, St. Andrews, NB, Canada EOG 2 X0. order to avoid this problem. A digitally encoded image does not suffer from mechanical jitter and is clean when sent to the frame-grabbing board. However, prices for these specialty VCRs are currently over $\$ 3,000$ US.

Another approach to image analysis is the superimposition of a video image on a computer monitor. The superimposition technique (Barnes, Vaughan, Jorgensen, \& Rosenbaum, 1989) involves lining up two monitors at $90^{\circ}$ and placing a half-silvered mirror at $45^{\circ}$ to the vertex between them, so that the video monitor is superimposed on the computer monitor. Unfortunately, there are a number of drawbacks to this system. First, the monitors have to be identical. If they have even slightly different curvatures, a problem with parallax will occur (Barnes et al., 1989). To correct for this problem, the observer must use one eye and place her/his head in the same location during digitization, or use a peep hole (Vaughan, personal communication, 1994) to ensure that the view is always the same and that any bias is systematic. The coordinates must then be transformed to correct for the difference between screens. It is preferable to use two identical multisync monitors $(2 \times \$ 400)$, but this will also make the superimposition technique expensive.

An inexpensive and easy-to-use alternative to either of the aforementioned techniques is to project a video image onto a digitizing tablet. Coordinates can then be captured by using a simple communication program to transfer data from the tablet with the PC. Digitization tablets have been used extensively as a tool by PC users. Durret (1980) outlined some of the digitizing tablets available in the late 1970 s. Since then, more tablets have become available, their resolution (1,000 lines/in.) and speed have improved, and their cost has decreased considerably. Today, tablets can be obtained for under $\$ 300$.

In this paper, we outline the basic optical theory and methodology to allow for construction of a videoprojection/digitization system. Also included are four example 
functions (programs) written in APL (APL*PLUS, STSC), to allow for input and capture of coordinates of the image sent from the digitizing tablet.

\section{Videoprojector}

Most of the optical theory required in order to build a videoprojector can be found in any college physics or engineering optics text (e.g., Blaker, 1971; Hecht, 1975; Meyer-Arendt, 1984; Williams \& Beckland, 1984). All that is needed to project an image from a video monitor to a surface is a bright monitor, a bellows, a lens (lenses), and a surface for projection. Variables involved in construction of the projector are (1) the object size (size of the monitor screen), (2) the image size (size of projected monitor image), and (3) the focal length of the lens/lenses.

The monitor picture (object) should be very bright, because brightness of the resulting image depends on the luminance of the image projected. As well, when the projected image is larger than the object, there is a proportional loss of image brightness. A bellows placed between the monitor and lens reduces light interference and maintains image brightness. For best results, the projector should be used in a dark room and the surface for projection should be a high-gain (reflective) material.

Image quality is also conditioned by the specifications of the lens chosen for projection. The simplest system consists of one thin biconvex lens which produces an inverted image. To create a righted image, one must turn the monitor upside down, use a monitor with an inverter switch, or invert the camera during recording. If the purpose of the projection system is to project the image onto a tablet, as in the system shown in Figure 1, the image will be righted after reflecting off the mirror. The choice of lens depends on size restrictions of the projector, object, and image. The Gaussian lens formula allows calculation of the appropriate focal length:

$$
\frac{1}{s_{o}}+\frac{1}{s_{i}}=\frac{1}{f},
$$

where $s_{o}$ is the distance from the lens to the object along the optical axis, $s_{i}$ is the distance from the lens to the image along the optical axis, and $f$ is the focal length of the lens.
The magnification factor is

$$
\text { magnification }=\frac{s_{i}}{s_{o}} \text {. }
$$

Equation 2 holds if the refractive indices of each side of the lens are equal (a symmetric biconvex lens). These two equations can be used to determine the focal length of lens required. Calculation of the parameters of construction of a projection system with an asymmetric biconvex lens is more complicated, but still possible (see Meyer-Arendt, 1984).

We recommend using a lens with a large diameter, because light transmittance from an object to an image is proportional to the lens diameter (or lens stop). Fresnel lenses are an inexpensive option if a large glass lens is not available. They consist of a series of concentric prisms (lines) mimicking the angle of curvature of a glass lens. The higher the density of lines, the closer a Fresnel lens approximates the image quality of a ground glass lens of the same focal length. A large-diameter (6-15 in.), high-resolution Fresnel lens costs less than \$35 US (Fresnel Optics, Inc., Rochester, NY). If accuracy and precision in measurement are not important, a Fresnel lens with a lower number of lines can be an extremely inexpensive option (under \$10 US).

As an alternative to what is discussed above, the projected image can be reinverted with a two-lens system. The following equations for two thin lenses will allow for the construction of a compound lens for projecting a righted image. Note that if the distance between lenses does not exceed the back focal length of the first lens, the image will not be reinverted.

$$
s_{i}=\frac{f_{2} d-\left(f_{1} f_{2} s_{o} /\left(s_{o}-f_{1}\right)\right)}{d-f_{2}-\left(f_{1} s_{o} /\left(s_{o}-f_{1}\right)\right)}
$$

and

$$
\text { magnification }=\frac{f_{1} s_{i}}{d\left(s_{o}-f_{1}\right)-s_{o} f_{1}},
$$

where $s_{i}$ is the distance from the first lens (lens closest to object) to the object; $s_{o}$ is the distance from the second lens to the image; $f_{1}$ and $f_{2}$ are the focal lengths of

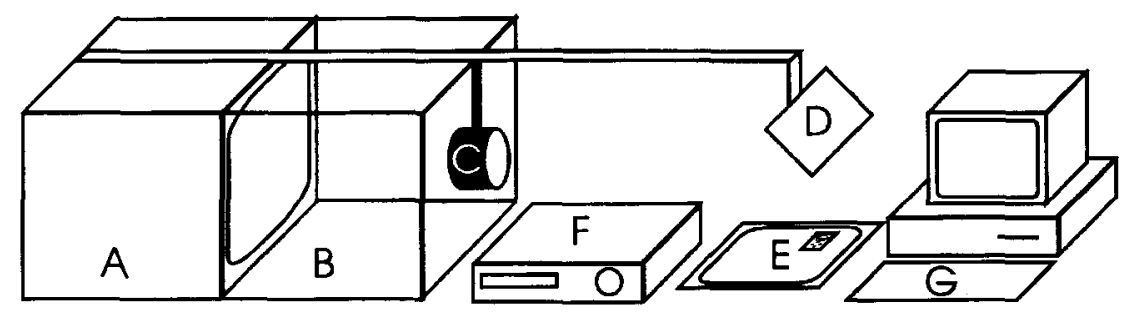

Figure 1. Videoprojection/digitization system: (A) monitor; (B) bellows; (C) lens; (D) mirror; (E) digitization tablet; (F) VCR; and (G) computer. 
the two lenses; and $d$ is the distance between the two lenses (Hecht, 1975).

The quality of videocamera and projection lenses used to record and project images may affect the accuracy of measurements. Both videocamera and projection lenses may be subject to aberrations (imperfections). Lens aberrations, such as spherical aberrations, coma, astigmatism, curvature of field, and chromatic aberrations can affect image quality (Williams \& Beckland, 1984). However, if distortions occur, they are often in the form of pin-cushioned or barrel distortions (Figure 2). We recommend that every image analysis system, regardless of type, should be calibrated for distortions by testing a grid pattern and modeling for imperfections. Coordinate data can be then transformed to correct for warpage of the coordinate plane. Chromatic aberrations can be mitigated by using achromatic lenses.

Another source of lenses which can lead to excellent results is to "borrow" a lens from an existing projection system. Opaque projectors are often fitted with lenses suitable for a videoprojection system. They generally have a large diameter and are sometimes achromatic lenses. The system shown in Figure 1 used an achromatic HS-Opaque 1000 projector lens manufactured by American Optical. It has a focal length of $46 \mathrm{~cm}$ and a diameter of $135 \mathrm{~mm}$.

\section{Capturing Coordinates for Behavioral Research}

For illustrative purposes, we have included a brief description of a system (Figure 1) that was developed to analyze the response of shoaling fish to the threat of aerial predation (Litvak, 1990, 1993). Litvak used this system to record positions of the head and tail of each fish from orthogonal pairs of views to allow for the reconstruction of their positions in three dimensions. Individual trajectories of swimming fish were traced on a white piece of paper taped to the digitizing tablet. The coordinates were digitized every second frame $(1 / 15$ th $\mathrm{sec})$. Fish identity was verified by shuttling back and forth through "frozen" images by using the VCR control. Because this videoprojection/digitization system was not linked directly to the computer, there was no problem with image synchronization between the VCR and the computer. Therefore, freeze-frame features of a VCR which did not have a digital signal processor could be used. In addition, using paper on the digitizing tablet also increased reflectance (image brightness) and provided a convenient place to write experimental notes. Litvak $(1990,1993)$ used this three-dimensional coordinate data to calculate individual fish trajectories through time, total displacement, depth, nearest neighbor distance, shoal shape in three dimensions, and shoal polarity (the degree to which fish in the shoal were aligned).

The accuracy of measurements made in reconstructing the positions of fish in three dimensions was tested by placing a test-tube rack in a variety of positions in the experimental aquarium $(61 \mathrm{~cm}$ long $\times 26 \mathrm{~cm}$ high $\times$ $21 \mathrm{~cm}$ wide). The ends of the tube supports were used as reference points for three-dimensional reconstruction. We used the videoprojection/digitization system to capture and record coordinates of the test positions. We took 135 distance measurements between the tips of the testtube stand. The real distance measures for the reference points were determined with a caliper. The mean absolute error of the reconstructed distances was determined to be $3.6 \mathrm{~mm}( \pm 0.031 \mathrm{SE})$. A $95 \%$ confidence interval was calculated for the mean absolute error, and the upper bound to this error was used to establish the limit of accuracy for the system. Distance measures of the system were accurate to $\pm 4.2 \mathrm{~mm}$ in three dimensions (Litvak 1990, 1993).

\section{Data Acquisition}

The function (note: APL programs are referred to as functions; see Listings) GRAB sets up communication between, in this example, a Summagraphics Digibitpad using a 4-button cross-hair mouse and a personal computer. GRAB assumes that the Summagraphics Digibitpad stream status (operating mode) is set to "B," which sends one pair of coordinates for each button pressed.

The functions DIGITIZER, INPUT, and DIGIT control the input and capture of the signals sent from the

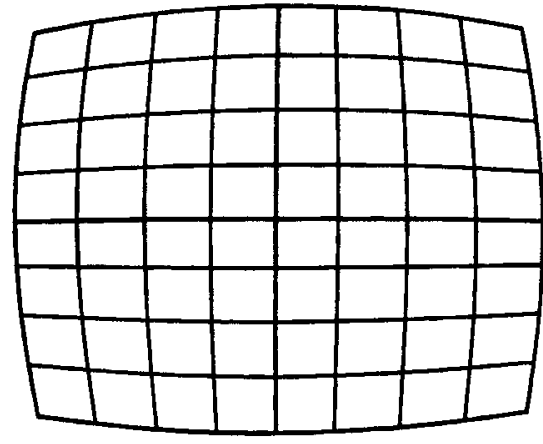

A

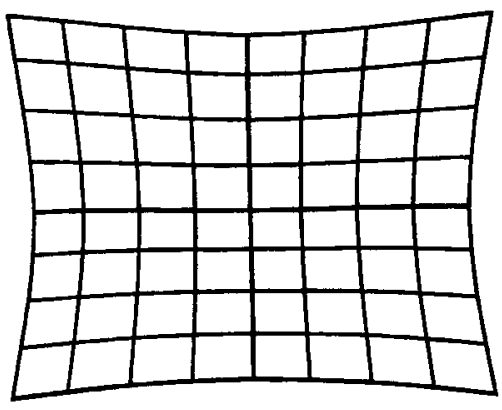

B

Figure 2. (A) Barrel distortion; (B) pin-cushioned distortion. 
digitizing pad. The function DIGITIZER is used to control acquisition of coordinates and assign them to an APL file. DIGITIZER allows the user to define the APL file name (or append the data to an existing file), write a note in the first component of the file as a header (file description), and choose the number of coordinates to be recorded in each file. Data are recorded with the first button on the digitizer's mouse. DIGITIZER prints the data to the screen as it is received from the Digibitpad. We have also added an error subroutine to allow the operator to correct the previous coordinates. To change a previously captured value, one uses mouse buttons 2,3 , or 4 . This will erase the previous value, and the correct coordinate can be entered with button 1 . After the last coordinate pair has been captured, the operator will be prompted to decide whether the current set of coordinates should be saved or discarded. If the data are to be saved, it is appended to the storage file. The operator will then be asked whether the session is to be terminated or another set of coordinates is to be recorded.

INPUT and DIGIT are subfunctions in DIGITIZER. INPUT controls the coordinate flow from the digitizing tablet to the PC. A bell is sounded when data are received by the computer through INPUT. DIGIT assigns the coordinates and the key numbers to their variables.

These functions can easily be modified to record extra information during digitizing. For example, descriptors of positional information can be added to the coordinate data by defining regions on the digitizing tablet. When the operator sends a signal from these preset zones, a descriptor is added to the data being sent from the tablet. This technique makes it possible to record behavioral observations with the coordinate data acquired from the digitizing tablet. All that is required is a simple modification of the functions DIGIT and DIGITIZER.

\section{REFERENCES}

Barnes, H. J., Vaughan, J., Jorgensen, M. J., \& Rosenbaum, D. A. (1989). A low-cost method for digitizing videotaped continuous movements on the Macintosh. Behavior Research Methods, Instruments, \& Computers, 21, 255-258.

BLAKER, J. W. (1971). Geometrical optics. New York: Marcell Dekker. DURRET, H. J. (1980). Inexpensive plotters and digitizers for psychological research and instruction. Behavior Research Methods \& Instrumentation, 12, 244-247.

Hеснт, E. (1975). Schaum's outline of theory and problems of optics. New York: McGraw-Hill.

LITVAK, M. K. (1990). Predator avoidance, foraging behaviour and social transmission of information in fish shoals. Unpublished doctoral dissertation, University of Toronto.

LiTVAK, M. K. (1993). Response of shoaling fish to the threat of aerial predation. Environmental Biology of Fishes, 36, 183-192.

MeYer-ARENDT, J. R. (1984). Introduction to classical and modern optics. Englewood Cliffs, NJ: Prentice-Hall.

Williams, C. S., \& BeCKLAND, O. A. (1984). Optics: A short course for engineers and scientists. Malabi, FL: Krieger. 
Listing

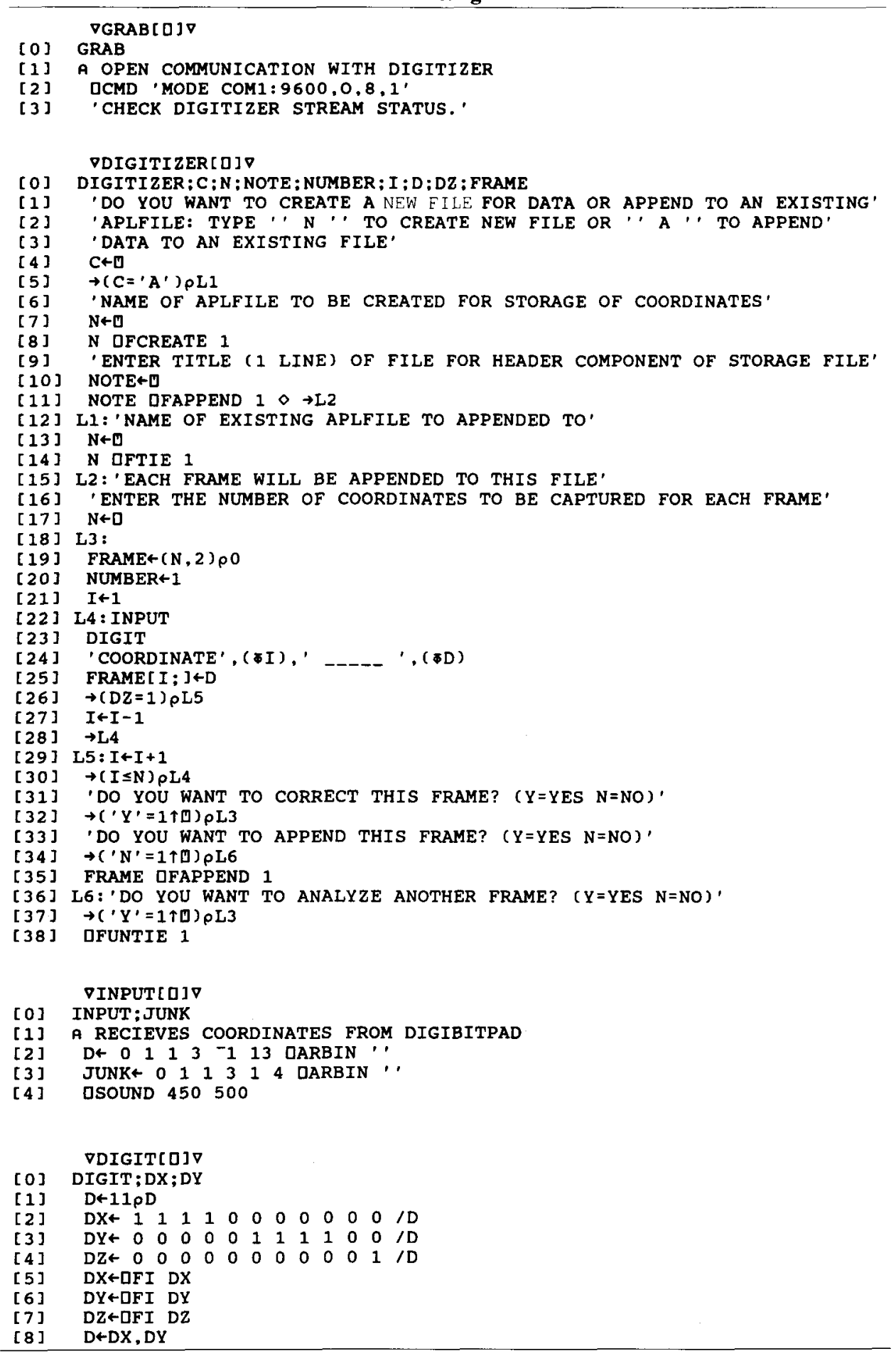

(Manuscript received January 20, 1994; revision accepted for publication July 18,1994 .) 